\title{
Spherical Distribution of 5 Points with Maximal Distance Sum
}

\author{
Xiaorong Hou $\cdot$ Junwei Shao
}

Received: 22 October 2009 / Revised: 7 August 2010 / Accepted: 6 October 2010 /

Published online: 21 October 2010

(C) Springer Science+Business Media, LLC 2010

\begin{abstract}
In this paper, we consider the problem of spherical distribution of 5 points, that is, how to configure 5 points on the unit sphere such that the mutual distance sum is maximal. It is conjectured that the sum of distances is maximal if the 5 points form a bipyramid distribution with two points positioned at opposite poles of the sphere and the other three positioned uniformly on the equator. We study this problem using interval methods and related techniques, and give a computer-assisted proof.
\end{abstract}

Keywords Spherical distribution · Interval analysis · Computer-assisted proof

\section{Introduction}

Studies on the problem of optimally arranging points on a sphere date back at least 100 years ago, when Thomson attempted to explain the periodic table in terms of the "plum pudding" model of the atom. Since then, several related problems have been proposed, some of which are still unsolved [11]. In general, these problems involve finding distributions of points on the surface of a sphere that maximize or minimize some quantity. In physics and chemistry, stable distributions are of interest, namely distributions that minimize some potential.

The problem has the following general form. Let $x_{1}, x_{2}, \ldots, x_{n}$ be points on the unit sphere $S^{m-1}$ of the Euclidean space $\mathbb{R}^{m}$ and set

Partially supported by a National Key Basic Research Project of China (2004CB318000) and by National Natural Science Foundation of China (61074189).

X. Hou $(\bowtie) \cdot$ J. Shao

School of Automation Engineering, University of Electronic Science and Technology of China, Sichuan, P.R. China

e-mail: houxr@uestc.edu.cn

J. Shao

e-mail: junweishao@gmail.com 


$$
V\left(X_{n}, m, \lambda\right)=\sum_{1 \leq i<j \leq n}\left|x_{i}-x_{j}\right|^{\lambda},
$$

where $X_{n}=\left\{x_{1}, x_{2}, \ldots, x_{n}\right\}$ and $\left|x_{i}-x_{j}\right|$ denotes the Euclidean distance between $x_{i}$ and $x_{j}$.

For $\lambda \leq 0$, let

$$
V_{1}(n, m, \lambda)=\min _{X_{n} \subset S^{m-1}} V\left(X_{n}, m, \lambda\right)
$$

where

$$
V_{1}(n, m, 0)=\min _{X_{n} \subset S^{m-1}} \sum_{1 \leq i<j \leq n} \log \frac{1}{\left|x_{i}-x_{j}\right|} .
$$

When $m=3$, this is the 7th Problem listed by Steve Smale in Mathematical Problems for the Next Century [26].

For $\lambda>0$, let

$$
V_{2}(n, m, \lambda)=\max _{X_{n} \subset S^{m-1}} V\left(X_{n}, m, \lambda\right) .
$$

So far, G. Pólya and G. Szegö [22] first studied problems of this type in the 1930s. Since then, a number of results about $V_{2}(n, m, \lambda)$ have been obtained. For example, L. Fejes Tóth [13] proved results for cases when $m=2, \lambda=1$ and when $n=m+1$, $\lambda=1$. E. Hille [18] considered the asymptotic properties of $V_{2}(n, m, \lambda) / n$ when $n \rightarrow \infty$ for specific $m$ and $\lambda$. K. B. Stolarsky proved bounds on $V_{2}(n, m, \lambda)$ for specific $m$ and $\lambda$ in $[28,29]$, and gave some properties of point distributions relating to $V_{2}(n, m, \lambda)$ when $m=2$ and $m=3$ in [30-32]. R. Alexander also proved bounds on $V_{2}(n, 3,1)$ in [1], and discussed some generalized sums of distances in [2, 3]. G.D. Chakerian and M.S. Klamkin proved bounds on $V_{2}(n, m, 1)$ in [9]. J. Berman and K. Hanes proved a property of the point distribution corresponding to $V_{2}(n, 3,1)$, and deduced some numerical results in [8]. G. Harman, J. Beck, T. Amdeberhan proved bounds on $V_{2}(n, m, \lambda)$ in $[4,7,17]$. A.V. Kolushov, V.A. Yudin and N.N. Andreev proved that, for $n=3$ and $\lambda=1$, the optimal configurations for $m=4,6,12$ are respectively the regular tetrahedron, octahedron, and icosahedron (for $\lambda=-1,0$, these configurations are also optimal $[5,6,21])$. Similar problems were also discussed in $[15,19,20,24]$.

Call the problem of calculating $V_{2}(n, m, \lambda)$ the $(n, m)$-problem. The case of five points in three dimensions (the $(5,3)$-problem) is of special significance. One of the most important recent attacks on general problems of this type is that of $\mathrm{H}$. Cohn and A. Kumar [10]. Their paper completely solves the $(n, m)$-problem (and, in fact, for any completely monotonic potential) provided a "sharp configuration" of $n$ points exists. Sharp configurations may exist for certain large values of $n=n(m)$, and they exist (regular $n$-gons) for all $(n, 2), n \geq 2$. For $(n, 3)$, the case $n=5$ is the smallest value of $n \geq 2$ for which no sharp configuration exists [12], hence the special significance of this case. For the Coulomb potential $\left(V_{1}(5,3,-1)\right)$ the details of a computer assisted proof have been posted by R.E. Schwartz [25]. It is conceivable that his framework could be modified to provide yet another computer-assisted proof of the present result.

It is remarkable that [12] proves, without any computer assistance, that $V_{1}(5,3,0)$ is achieved by the present bipyramidal distribution. 
Fig. 1 The spherical coordinate system

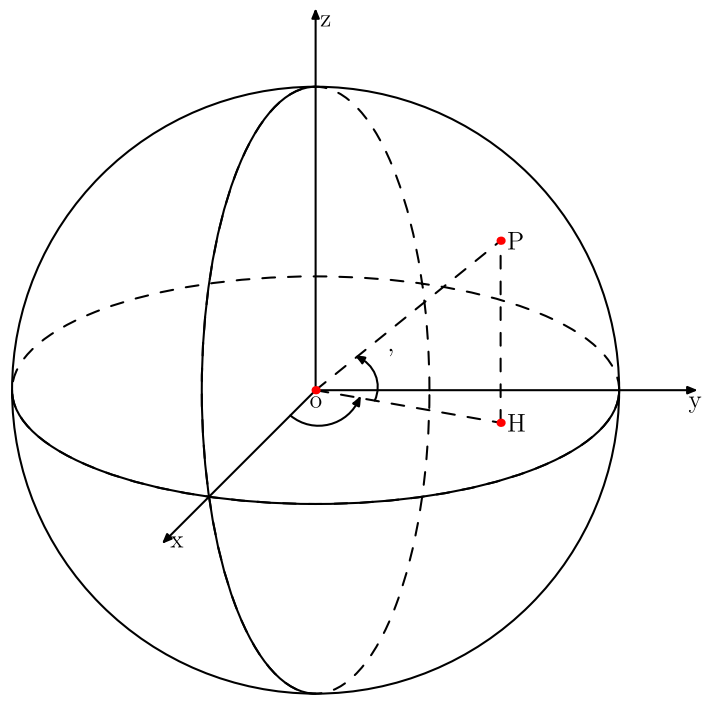

In this paper, we solve the $(5,3)$-problem for $\lambda=1$. We show that this maximum is uniquely achieved by the bipyramidal distribution in which two of the points are at the opposite poles, and the remaining three points are uniformly distributed around the equator (an obvious and long-standing conjecture). The proof rests upon computer implementation of interval analysis.

The main idea of our proof is as follows. Firstly, we express $V\left(X_{5}, 3,1\right)$ as a function in a certain coordinate system; secondly, we exclude a domain where the bipyramid distribution corresponds to the only maximum of $V\left(X_{5}, 3,1\right)$; and finally, we subdivide the remaining domain and prove that points in these subdomains cannot be global maximum of the function. This establishes the conjecture.

\section{Mathematical Descriptions of the Problem}

\subsection{Spherical Coordinate System}

We choose the spherical coordinate system as shown in Fig. 1. A point $P$ on $S^{2}$ is identified by $(1, \phi, \theta)$, where $\phi \in\left[-\frac{\pi}{2}, \frac{\pi}{2}\right]$ is the angle from vector $\overrightarrow{O H}$, i.e., the projection of vector $\overrightarrow{O P}$ in $x o y$-plane, to vector $\overrightarrow{O P}$, positive if the $z$-coordinate of $P$ is positive, and $\theta \in[-\pi, \pi)$ is the angle from $x$-axis to vector $\overrightarrow{O H}$, positive if the $y$-coordinate of $P$ is positive.

According to such definitions, we have the following formulas transforming from spherical coordinates $(1, \phi, \theta)$ to Cartesian coordinates $(x, y, z)$,

$$
\left\{\begin{array}{l}
x=\cos (\phi) \cos (\theta), \\
y=\cos (\phi) \sin (\theta), \\
z=\sin (\phi) .
\end{array}\right.
$$


Considering the spherical symmetry, we can choose the spherical coordinates for 5 points as follows:

$$
A(1,0,0), B\left(1, \phi_{1}, \pi\right), C\left(1, \phi_{2}, \theta_{2}\right), D\left(1, \phi_{3}, \theta_{3}\right), E\left(1, \phi_{4}, \theta_{4}\right)
$$

where

$$
\begin{aligned}
\Theta & \triangleq\left(\phi_{1}, \phi_{2}, \theta_{2}, \phi_{3}, \theta_{3}, \phi_{4}, \theta_{4}\right) \\
\in \mathcal{D} & \triangleq\left(\left[-\frac{\pi}{2}, \frac{\pi}{2}\right],\left[-\frac{\pi}{2}, \frac{\pi}{2}\right],[-\pi, \pi),\left[-\frac{\pi}{2}, \frac{\pi}{2}\right],[-\pi, \pi),\left[-\frac{\pi}{2}, \frac{\pi}{2}\right],[-\pi, \pi)\right),
\end{aligned}
$$

then the sum of mutual distances of the above points is

$$
\begin{aligned}
f(\Theta)= & \sqrt{2+2 \cos \left(\phi_{1}\right)}+\sqrt{2-2 \cos \left(\phi_{2}\right) \cos \left(\theta_{2}\right)} \\
& +\sqrt{2-2 \cos \left(\phi_{3}\right) \cos \left(\theta_{3}\right)}+\sqrt{2-2 \cos \left(\phi_{4}\right) \cos \left(\theta_{4}\right)} \\
& +\sqrt{2 \cos \left(\phi_{1}\right) \cos \left(\phi_{2}\right) \cos \left(\theta_{2}\right)+2-2 \sin \left(\phi_{1}\right) \sin \left(\phi_{2}\right)} \\
& +\sqrt{2 \cos \left(\phi_{1}\right) \cos \left(\phi_{3}\right) \cos \left(\theta_{3}\right)+2-2 \sin \left(\phi_{1}\right) \sin \left(\phi_{3}\right)} \\
& +\sqrt{2 \cos \left(\phi_{1}\right) \cos \left(\phi_{4}\right) \cos \left(\theta_{4}\right)+2-2 \sin \left(\phi_{1}\right) \sin \left(\phi_{4}\right)} \\
& +\sqrt{-2 \cos \left(\phi_{3}\right) \cos \left(\phi_{2}\right) \cos \left(\theta_{2}-\theta_{3}\right)+2-2 \sin \left(\phi_{2}\right) \sin \left(\phi_{3}\right)} \\
& +\sqrt{-2 \cos \left(\phi_{2}\right) \cos \left(\phi_{4}\right) \cos \left(\theta_{2}-\theta_{4}\right)+2-2 \sin \left(\phi_{2}\right) \sin \left(\phi_{4}\right)} \\
& +\sqrt{-2 \cos \left(\phi_{3}\right) \cos \left(\phi_{4}\right) \cos \left(\theta_{3}-\theta_{4}\right)+2-2 \sin \left(\phi_{3}\right) \sin \left(\phi_{4}\right)}
\end{aligned}
$$

The Hessian matrix of $f$ is denoted by

$$
H(\Theta)=\left(\frac{\partial^{2} f}{\partial \Theta_{i} \partial \Theta_{j}}\right)_{7 \times 7} .
$$

\subsection{Bipyramid Distribution}

There is a $\Theta$ in $\mathcal{D}$ corresponding to a bipyramid distribution, that is,

$$
\Theta_{b p}=\left(-\frac{\pi}{3}, \frac{\pi}{3}, \pi, 0,-\frac{\pi}{2}, 0, \frac{\pi}{2}\right),
$$

as shown in Fig. 2.

We have

$$
\begin{aligned}
f\left(\Theta_{b p}\right) & =3 \sqrt{3}+6 \sqrt{2}+2 \\
& \approx 15.68143380
\end{aligned}
$$


Fig. 2 The bipyramid distribution

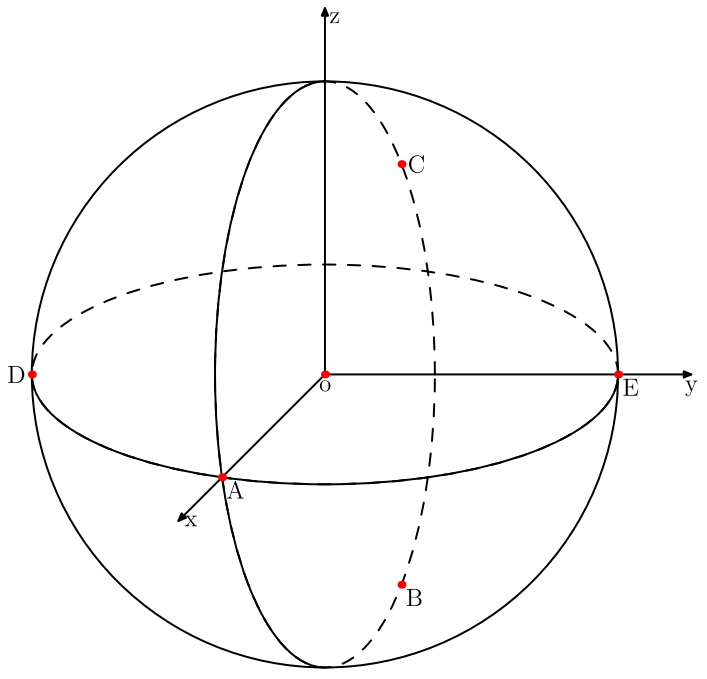

and

$$
H\left(\Theta_{b p}\right)=\left(\begin{array}{ccccccc}
\frac{-\sqrt{3}}{2} & \frac{\sqrt{3}}{4} & 0 & \frac{-\sqrt{2}}{4} & \frac{\sqrt{6}}{4} & \frac{-\sqrt{2}}{4} & \frac{-\sqrt{6}}{4} \\
\frac{\sqrt{3}}{4} & \frac{-\sqrt{3}}{2} & 0 & \frac{-\sqrt{2}}{4} & \frac{-\sqrt{6}}{4} & \frac{-\sqrt{2}}{4} & \frac{\sqrt{6}}{4} \\
0 & 0 & \frac{-2 \sqrt{3}-3 \sqrt{2}}{24} & \frac{-\sqrt{6}}{16} & \frac{\sqrt{2}}{16} & \frac{\sqrt{6}}{16} & \frac{\sqrt{2}}{16} \\
\frac{-\sqrt{2}}{4} & \frac{-\sqrt{2}}{4} & \frac{-\sqrt{6}}{16} & \frac{-3 \sqrt{2}-4}{8} & 0 & \frac{-1}{2} & 0 \\
\frac{\sqrt{6}}{4} & \frac{-\sqrt{6}}{4} & \frac{\sqrt{2}}{16} & 0 & \frac{-3 \sqrt{2}-4}{8} & 0 & \frac{1}{2} \\
\frac{-\sqrt{2}}{4} & \frac{-\sqrt{2}}{4} & \frac{\sqrt{6}}{16} & \frac{-1}{2} & 0 & \frac{-3 \sqrt{2}-4}{8} & 0 \\
\frac{-\sqrt{6}}{4} & \frac{\sqrt{6}}{4} & \frac{\sqrt{2}}{16} & 0 & \frac{1}{2} & 0 & \frac{-3 \sqrt{2}-4}{8}
\end{array}\right) .
$$

The matrix $H\left(\Theta_{b p}\right)$ is negative definite, so the bipyramid distribution corresponds to a maximum of $f$.

\subsection{Mathematical Description}

As a matter of fact, what we are to prove is

Conjecture 1 Without considering spherical symmetry, the function $f(\Theta)$ in (2.4) has the unique maximum point $\Theta_{b p}$ in $\mathcal{D}$.

In the remaining part of this paper, we will prove this conjecture according to following outlines.

1. Giving some results to demonstrate that we only need to analyze $f$ over a domain contained in $\mathcal{D}$, i.e., $\mathcal{D}^{(1)} \cup \mathcal{D}^{(2)}$ (see Conjecture 2).

2. Analyzing interval Hessian matrices (Theorems 4.3 and 4.5) to prove that $\Theta_{b p}$ is the unique local maximum point of $f$ in its neighborhood $\mathcal{D}_{b p} \subseteq \mathcal{D}^{(1)} \cup \mathcal{D}^{(2)}$, where $\stackrel{\circ}{\mathcal{D}}_{b p}$ denotes the interior of $\mathcal{D}_{b p}$ (see Proposition 4.1). 
3. Analyzing interval Hessian matrices (Theorems 4.4 and 4.6) to prove that $f$ cannot attain its maximum in a subdomain of $\mathcal{D}^{(1)} \cup \mathcal{D}^{(2)}$, i.e., $\mathcal{D}_{p}$ (see Proposition 5.1).

4. Making use of related results and interval computation (Sect. 4.1.1) to prove that in the remaining domain, i.e., $\left(\mathcal{D}^{(1)} \cup \mathcal{D}^{(2)}\right) \backslash\left(\mathcal{D}_{b p} \cup \mathcal{D}_{p}\right)$, points are spherically symmetric to $\Theta_{b p}$, or not maximum points of $f$ in $\mathcal{D}^{(1)} \cup \mathcal{D}^{(2)}$ (see Sect. 6.3).

\section{Simplified Problem}

\subsection{Some Results}

We need following results so as to simplify the problem.

Proposition 3.1 If some distribution of 5 points corresponds to larger distance sum than $f\left(\Theta_{b p}\right)$, and $A B$ is the second largest distance among $\left(\begin{array}{l}5 \\ 2\end{array}\right)=10$ distances, $\phi_{1}$ should satisfy

$$
\phi_{1} \geq-2 \arccos (\sqrt{3} / 6+\sqrt{2} / 3)
$$

Proof From (2.7), we know that in order to attain a larger distance sum than $f\left(\Theta_{b p}\right)$, the second largest distance must be not smaller than

$$
((3 \sqrt{3}+6 \sqrt{2}+2)-2) / 9=\sqrt{3} / 3+2 \sqrt{2} / 3 .
$$

With the condition that $A B$ is the second largest distance, the result required can be deduced immediately.

Proposition 3.2 If 5 points are on the same half sphere, $f$ cannot attain its global maximum.

Proof Without loss of generality, suppose the $z$-coordinates of 5 points are all nonnegative. If the $z$-coordinate of some point is positive, we move it to the symmetric position with respect to the xoy-plane, then we will get a larger distance sum. If 5 points are all distributed on the $x o y$-plane, the maximal distance sum is $5 \cot \frac{\pi}{10}(5$ points form a regular pentagon [13]), which is obviously smaller than $f\left(\Theta_{b p}\right)$.

Proposition 3.3 If a partial derivative of the function $f$ does not vary signs in a domain, then there exists no critical point of $f$ in this domain.

Theorem 3.1 [30] Suppose 5 points are placed so that $f$ is maximal, then any distance between two points cannot be less than $\frac{2}{15}$.

Theorem 3.2 [8] Let $p_{1}, \ldots, p_{n}$ be points on the unit sphere $S^{2}$ in $\mathbb{R}^{3}, f: S^{2} \rightarrow \mathbb{R}$ be a function defined as $f(x)=\sum_{i=1}^{n}\left|x-p_{i}\right|$. If $f$ has a maximum at $p$, then $p=$ $q /|q|$, where $q=\sum_{i=1}^{n}\left(p-p_{i}\right) /\left|p-p_{i}\right|$. 


\subsection{Some Assumptions}

In order to find a distribution with the distance sum larger than $f\left(\Theta_{b p}\right)$, we can make the following assumptions.

Assumption 3.1 AB is the second largest distance among $\left(\begin{array}{l}5 \\ 2\end{array}\right)=10$ distances.

Assumption 3.2 (By the spherical symmetry) $D$ is on the left-half sphere (i.e., $\left.y_{D} \leq 0\right), C, E$ are on the right-half sphere (i.e., $\left.y_{C}, y_{E} \geq 0\right), C$ is above $E$ (i.e., $\left.z_{C} \geq z_{E}\right)$.

Assumption 3.3 (By Proposition 3.1) $\phi_{1} \geq-2 \arccos (\sqrt{3} / 6+\sqrt{2} / 3)$.

Assumption 3.4 (By Proposition 3.2) Five points are not on any half sphere.

Assumption 3.5 (By Theorem 3.1) Any distance between two points is larger than $\frac{2}{15}$.

Under these assumptions, the bipyramid distribution (corresponding to the maximal distance sum conjectured) and the pyramid distribution (corresponding to another critical point of $f$ ) each corresponds to a unique $\Theta$. Furthermore, we only need to consider $f(\Theta)$ over the following two subdomains of $\mathcal{D}$ according to Assumptions 3.1-3.3.

1. $D$ is on the upper-half sphere (denote this domain by $\mathcal{D}^{(1)}$ ):

$$
\begin{aligned}
\phi_{1} & \in[-2 \arccos (\sqrt{3} / 6+\sqrt{2} / 3), 0], \\
\phi_{2} & \in[-\pi / 2,0], \\
\theta_{2} & \in[0, \pi], \\
\phi_{3} & \in[0, \pi / 2], \\
\theta_{3} & \in[-\pi, 0], \\
\phi_{4} & \in[-\pi / 2,0], \\
\theta_{4} & \in[0, \pi] .
\end{aligned}
$$

2. $D$ is on the lower-half sphere, $C$ is on the upper-half sphere (denote this domain by $\left.\mathcal{D}^{(2)}\right)$ :

$$
\begin{aligned}
\phi_{1} & \in[-2 \arccos (\sqrt{3} / 6+\sqrt{2} / 3), 0], \\
\phi_{2} & \in[0, \pi / 2], \\
\theta_{2} & \in[0, \pi], \\
\phi_{3} & \in[-\pi / 2, \pi / 2], \\
\theta_{3} & \in[-\pi, 0], \\
\phi_{4} & \in[-\pi / 2, \pi / 2], \\
\theta_{4} & \in[0, \pi] .
\end{aligned}
$$


Now, we are to prove the following conjecture which is equivalent to Conjecture 1.

Conjecture 2 Under Assumptions 3.1-3.5, $f(\Theta)$ attains its unique global maximum in $\mathcal{D}^{(1)} \cup \mathcal{D}^{(2)}$ at $\Theta_{b p}$.

\section{Domain Near Coordinates Corresponding to the Bipyramid Distribution}

\subsection{Interval Methods}

We first briefly introduce the interval methods we used in our proof.

\subsubsection{Interval Computation}

An interval is defined as a set [27]:

$$
X=[a, b]=\{x: a \leq x \leq b\},
$$

where $a, b \in \mathbb{R} . \underline{X}, \bar{X}$ respectively denote the left and right vertices of the interval $X$.

For intervals $X$ and $Y$, if $x>y$ for each $x \in X$ and each $y \in Y$, we say that $X>Y$. Other interval relations are understood the same way. An $n$-tuple of intervals $\mathbf{X}=\left(X_{1}, \ldots, X_{n}\right)$ can be used to denote some rectangular domain in $\mathbb{R}^{n}$. Let $\mathbb{R}$ be the set of intervals over $\mathbb{R}$, and $\mathbb{R} \mathbb{R}^{n}$ be the set of $n$-tuples of intervals.

There exists an imbedding from $\mathbb{R}$ to $\mathbb{I R}$ defined as follows

$$
\mu(x)=[x, x]
$$

thus for numbers in $\mathbb{R}$, we can also consider them as intervals.

Interval arithmetic over $\mathbb{I R}$ is defined as

$$
X \circ Y=\{x \circ y: x \in X, y \in Y\},
$$

where $\circ$ is "+, “-", “*” or " /", and for $Y$ containing 0 , we prescribe that $X / Y$ is the smallest interval containing $\{x / y: x \in X, y \in Y \backslash\{0\}\}$. Furthermore, for an elementary function $f$, a corresponding elementary interval mapping can be defined as

$$
f(X)=\{f(x): x \in X\} .
$$

When operands of interval arithmetic or arguments of elementary functions are intervals, we consider underlying computations as interval computations defined above, and the interval computation is of the same precedence as the corresponding arithmetical computation.

Under above definitions, an arbitrary elementary function $f: \mathbb{R}^{n} \rightarrow \mathbb{R}$ can be expanded to a mapping over $\mathbb{I} \mathbb{R}^{n} \rightarrow \mathbb{I} \mathbb{R}$ :

$$
\tilde{f}(\mathbf{X})=f(\mathbf{X})
$$


Through such $\tilde{f}$, we can get an interval which contains the range of $f$ over rectangular domain $\mathbf{X}$.

As a matter of fact, there are related programs used to process interval computation, such as the procedure evalr in the Maple system which can be used to implement interval computation without errors. But in practice, errorlessness is not necessary, what we need are just intervals containing the ranges of functions over domains. Another problem is that performing such errorless interval computation is always time-consuming, thus it cannot meet our needs.

Considering the efficiency and the accuracy, we wrote a package IntervalArithmetic based on the Maple system. The package uses rational numbers as interval vertices, thus the computational result of $f(X)$ is slightly different from its definition in (4.3), i.e., the package uses an enlarged rational interval to represent the interval $\{f(x): x \in X\}$. In the end, the computational result of $\tilde{f}(\mathbf{X})$ is also a larger rational interval containing its definition in (4.4); however, the difference can reduce to zero as intervals of $\mathbf{X}$ shrink to points, and the variable "Digits" in the Maple system which controls the number of significant digits in software floating-point computations becomes infinity.

The following example may be considered as a simple illustration about how we use interval computation in our computer-assisted proof.

Example $4.1 \mathcal{D}_{1}^{(2)}=\left(\Phi_{1}, \Phi_{2}, \Theta_{2}, \Phi_{3}, \Theta_{3}, \Phi_{4}, \Theta_{4}\right)$ is a rectangular domain contained in $\mathcal{D}^{(2)}$, where

$$
\begin{aligned}
& \Phi_{1}=\left[-\frac{70736064244757919}{50000000000000000},-\frac{23578688081585973}{25000000000000000}\right], \\
& \Phi_{2}=\left[-\frac{7853981633974483097}{5000000000000000000},-\frac{7853981633974483097}{7500000000000000000}\right], \\
& \Theta_{2}=\left[0, \frac{5235987755982988731}{5000000000000000000}\right], \\
& \Phi_{3}=\left[0, \frac{7853981633974483097}{15000000000000000000}\right], \\
& \Theta_{3}=\left[-\frac{31415926535897932387}{10000000000000000000},-\frac{31415926535897932387}{15000000000000000000}\right], \\
& \Phi_{4}=\left[-\frac{7853981633974483097}{5000000000000000000},-\frac{7853981633974483097}{7500000000000000000}\right], \\
& \Theta_{4}=\left[0, \frac{5235987755982988731}{5000000000000000000}\right] .
\end{aligned}
$$

Suppose $\tilde{g}(\mathbf{X})$ is the expanded mapping corresponding to $g(\Theta)=f(\Theta)-f\left(\Theta_{b p}\right)$, as is defined in (4.4). Following the rules prescribed above, we can calculate that 


$$
\begin{aligned}
& \cos \left(\Phi_{1}\right)=\left[\frac{15544216366986277489}{100000000000000000000}, \frac{14681082495913117063}{25000000000000000000}\right], \\
& 2 \cos \left(\Phi_{1}\right)=\left[\frac{15544216366986277489}{50000000000000000000}, \frac{14681082495913117063}{12500000000000000000}\right], \\
& 2 \cos \left(\Phi_{1}\right)+2=\left[\frac{115544216366986277489}{50000000000000000000}, \frac{39681082495913117063}{12500000000000000000}\right], \\
& \sqrt{2 \cos \left(\Phi_{1}\right)+2}=\left[\frac{760079654927647295278245640930483049783}{500000000000000000000000000000000000000},\right. \\
& \vdots \\
& \sqrt{-2 \cos \left(\Phi_{3}\right) \cos \left(\Phi_{4}\right) \cos \left(\Theta_{3}-\Theta_{4}\right)+2-2 \sin \left(\Phi_{3}\right) \sin \left(\Phi_{4}\right)} \\
& =\left[\frac{11048543456039805064938434576854386221}{7812500000000000000000000000000000000},\right. \\
& \left.\frac{2500000000000000000170806153595479857903}{1250000000000000000000000000000000000000}\right],
\end{aligned}
$$

Step by step, finally we can get

$$
\begin{aligned}
\tilde{g}\left(\mathcal{D}_{1}^{(2)}\right) & =\left[-\frac{64195023638006381129}{10000000000000000000},-\frac{12440205351457241497}{25000000000000000000}\right] \\
& \subseteq[-6.4196,-.4976],
\end{aligned}
$$

thus $f(\Theta)-f\left(\Theta_{b p}\right)<0$ when $\Theta$ takes values in $\mathcal{D}_{1}^{(2)}$.

\subsubsection{Interval Matrices}

Relations of real matrices of the same order are understood componentwise. An interval matrix is defined as the following set of matrices:

$$
\left(\left[\underline{a}_{i j}, \bar{a}_{i j}\right]\right)=[\underline{A}, \bar{A}]=\left\{A \in \mathbb{R}^{n \times n}: \underline{A} \leq A \leq \bar{A}\right\},
$$

where

$$
\underline{A}=\left(\underline{a}_{i j}\right), \quad \bar{A}=\left(\bar{a}_{i j}\right) .
$$


When $\underline{A}$ and $\bar{A}$ are symmetric, we call the set of symmetric matrices in $[\underline{A}, \bar{A}]$ a symmetric interval matrix which is also denoted by $[\underline{A}, \bar{A}]$.

For a interval matrix $[\underline{A}, \bar{A}]$, denote its midpoint matrix by $A_{c}=\frac{A+\bar{A}}{2}$, and its radius matrix by $A_{\delta}=\frac{\bar{A}-\underline{A}}{2}$. For a real symmetric matrix $A$, it is well know that all its eigenvalues are real, we denote them in decreasing order by $\lambda_{1}(A) \geq$ $\lambda_{2}(A) \geq \cdots \geq \lambda_{n}(A)$, and denote the spectral radius of $A$ (i.e., the maximum eigenvalue modulus) by $\rho(A)$. For bounds on eigenvalues of matrices in an interval matrix, it can be directly deduced from the Wielandt-Hoffman theorem [16] that

Theorem 4.1 For a symmetric interval matrix $[\underline{A}, \bar{A}]$, the set

$$
\left\{\lambda_{i}(A): A \in[\underline{A}, \bar{A}]\right\}
$$

is a compact interval, denote this compact interval by

$$
\left[\underline{\lambda}_{i}([\underline{A}, \bar{A}]), \bar{\lambda}_{i}([\underline{A}, \bar{A}])\right], \quad 1 \leq i \leq n,
$$

then

$$
\left[\underline{\lambda}_{i}([\underline{A}, \bar{A}]), \bar{\lambda}_{i}([\underline{A}, \bar{A}])\right] \subseteq\left[\lambda_{i}\left(A_{c}\right)-\rho\left(A_{\delta}\right), \lambda_{i}\left(A_{c}\right)+\rho\left(A_{\delta}\right)\right], \quad i=1, \ldots, n .
$$

In fact, $\bar{\lambda}_{1}([\underline{A}, \bar{A}])$ and $\underline{\lambda}_{n}([\underline{A}, \bar{A}])$ can be found explicitly [23], that is,

Theorem 4.2 A real symmetric interval matrix

$$
\left(\left[\underline{a}_{i j}, \bar{a}_{i j}\right]\right)=\left\{A \in \mathbb{R}^{n \times n}: \underline{A} \leq A \leq \bar{A}, \underline{A}=\left(\underline{a}_{i j}\right), \bar{A}=\left(\bar{a}_{i j}\right)\right\}
$$

corresponds to the following $2^{n-1}$ vertex matrices:

$$
A_{k}=\left(a_{k i j}\right), \quad 0 \leq k \leq 2^{n-1}-1,
$$

where we denote the binary representation for $k$ by $k=\left(k_{1} k_{2} \cdots k_{n}\right)_{2}$, and

$$
a_{k i j}=\frac{1}{2}\left(\underline{a}_{i j}+\bar{a}_{i j}+(-1)^{k_{i}+k_{j}}\left(\underline{a}_{i j}-\bar{a}_{i j}\right)\right) .
$$

For matrices in this symmetric interval matrix, the minimal (or maximal) eigenvalues of them attain the minimum (respectively, maximum) at some vertex matrix $A_{k}$.

For a real symmetric interval matrix $[\underline{A}, \bar{A}],[\underline{A}, \bar{A}] \succ 0([\underline{A}, \bar{A}] \succeq 0)$ means that $A$ is positive (semi)definite for each $A \in[\underline{A}, \bar{A}]$, and $[\underline{A}, \bar{A}] \nsucc 0([\underline{A}, \bar{A}] \nsucceq 0)$ means that $A$ is not positive (semi)definite for each $A \in[\underline{A}, \bar{A}]$. Symbols $\prec, \preceq, \nprec, \npreceq$ can be defined similarly.

From Theorem 4.2, we have the following necessary and sufficient criterion for determining whether a symmetric interval matrix $[\underline{A}, \bar{A}]$ satisfies $[\underline{A}, \bar{A}] \succ 0$. 
Theorem 4.3 The real symmetric interval matrix

$$
\left(\left[\underline{a}_{i j}, \bar{a}_{i j}\right]\right)=\left\{A \in \mathbb{R}^{n \times n}: \underline{A} \leq A \leq \bar{A}, \underline{A}=\left(\underline{a}_{i j}\right), \bar{A}=\left(\bar{a}_{i j}\right)\right\}
$$

satisfies $\left(\left[\underline{a}_{i j}, \bar{a}_{i j}\right]\right) \succ 0$ if and only if the following $2^{n-1}$ vertex matrices are all positive definite:

$$
A_{k}=\left(a_{k i j}\right), \quad 0 \leq k \leq 2^{n-1}-1,
$$

where $A_{k}$ is defined as in Theorem 4.2 .

From Theorem 4.1, we have the following sufficient criterion for determining whether a symmetric interval matrix $[\underline{A}, \bar{A}]$ satisfies $[\underline{A}, \bar{A}] \nsucceq 0$.

Theorem 4.4 Suppose $[\underline{A}, \bar{A}]$ is a symmetric interval matrix, then $[\underline{A}, \bar{A}] \nsucceq 0$ if

$$
\lambda_{n}\left(A_{c}\right)+\rho\left(A_{\delta}\right)<0
$$

where $A_{c}$ and $A_{\delta}$ are the midpoint matrix and the radius matrix of $[\underline{A}, \bar{A}]$, respectively.

With the help of the above theorems, we can use the following results to determine extreme points of a function in a domain.

Theorem 4.5 [14] Suppose $K$ is an open, convex set in $\mathbb{R}^{n}, f \in C^{2}(K), \mathbf{x}_{0} \in K$ is a critical point of $f$, and the Hessian matrix $H(\mathbf{x})$ of $f$ is positive definite for all $\mathbf{x} \in K$, then $\mathbf{x}_{0}$ is the unique minimum point of $f$ in $K$.

Theorem 4.6 [14] Suppose $K$ is an open, convex set in $\mathbb{R}^{n}, f \in C^{2}(K)$, and the Hessian matrix $H(\mathbf{x})$ of $f$ is not positive semidefinite for all $\mathbf{x} \in K$, then there exists no minimum point of $f$ in $K$.

\subsection{Neighborhood of $\Theta_{b p}$}

Now we introduce a perturbation $\left[-\frac{\pi}{377}, \frac{\pi}{377}\right]$ on coordinates of $\Theta_{b p}$, and obtain a rectangular domain, i.e.,

$$
\left(\begin{array}{l}
\phi_{1} \\
\phi_{2} \\
\theta_{2} \\
\phi_{3} \\
\theta_{3} \\
\phi_{4} \\
\theta_{4}
\end{array}\right) \in\left(\begin{array}{c}
{\left[-\frac{380}{1131} \pi,-\frac{374}{1131} \pi\right]} \\
{\left[\frac{374}{1131} \pi, \frac{380}{1131} \pi\right]} \\
{\left[\frac{376}{377} \pi, \frac{378}{377} \pi\right]} \\
{\left[-\frac{1}{377} \pi, \frac{1}{377} \pi\right]} \\
{\left[-\frac{379}{754} \pi,-\frac{375}{754} \pi\right]} \\
{\left[-\frac{1}{377} \pi, \frac{1}{377} \pi\right]} \\
{\left[\frac{375}{754} \pi, \frac{379}{754} \pi\right]}
\end{array}\right) .
$$


In this domain, $\theta_{2}$ varies in $\left[\frac{376}{377} \pi, \frac{378}{377} \pi\right]$, which exceeds the bound we prescribed for $\theta_{2}$ in (2.3). But due to the periodicity of $f$, it is of no error. In fact, interval vertices are represented by rational numbers in the Maple package IntervalArithmetic, so these intervals whose vertices contain $\pi$ are enlarged to their rational representations. We denote this rectangular domain by $\mathcal{D}_{b p}$.

The interval Hessian matrix $\mathcal{V}$ of $f$ over $\mathcal{D}_{b p}$ can be calculated by interval computation. Through Theorem 4.3, we can judge that the symmetric interval matrix $\mathcal{V}$ satisfies $\mathcal{V} \prec 0$, and by Theorem 4.5 , the bipyramid distribution indeed corresponds to the maximum of $f$ in $\stackrel{\circ}{\mathcal{D}}_{b p}$, that is, we have

Proposition 4.1 $\Theta_{b p}$ is the unique local maximum point of $f$ in $\stackrel{\circ}{\mathcal{D}}_{b p}$.

\section{Domain Near Coordinates Corresponding to the Pyramid Distribution}

Under the assumptions in Sect. 3.2, the coordinates representing the pyramid distribution are unique. Since they correspond to a critical point of $f$, and the value of $f$ at this point is too close to $f\left(\Theta_{b p}\right)$, we discuss it separately.

5.1 Pyramid Distribution

If $\Theta \in \mathcal{D}^{(1)} \cup \mathcal{D}^{(2)}$, the pyramid distribution is unique, i.e.,

$$
\begin{aligned}
& A(1,0,0), \\
& B\left(1,-2 \omega_{1}, \pi\right), \\
& C\left(1, \frac{\pi}{2}-\omega_{1}, \pi\right), \\
& D\left(1, \omega_{2},-\omega_{3}\right), \\
& E\left(1, \omega_{2}, \omega_{3}\right),
\end{aligned}
$$

where

$$
\begin{aligned}
\omega_{1}= & \arcsin \left(-\frac{3}{4}+\frac{\sqrt{2}}{2}+\frac{\sqrt{41-28 \sqrt{2}}}{4}\right), \\
\omega_{2}= & -\arcsin \left(\left(-\frac{3}{4}+\frac{\sqrt{2}}{2}+\frac{\sqrt{41-28 \sqrt{2}}}{4}\right)\right. \\
& \left.\times \sqrt{1-\left(-\frac{3}{4}+\frac{\sqrt{2}}{2}+\frac{\sqrt{41-28 \sqrt{2}}}{4}\right)^{2}}\right), \\
\omega_{3}= & \operatorname{arccot}\left(\frac{\left(-\frac{3}{4}+\frac{\sqrt{2}}{2}+\frac{\sqrt{41-28 \sqrt{2}})^{2}}{4}\right.}{\left.\sqrt{1-\left(-\frac{3}{4}+\frac{\sqrt{2}}{2}+\frac{\sqrt{41-28 \sqrt{2}}}{4}\right.}\right)^{2}}\right),
\end{aligned}
$$


Fig. 3 The pyramid distribution

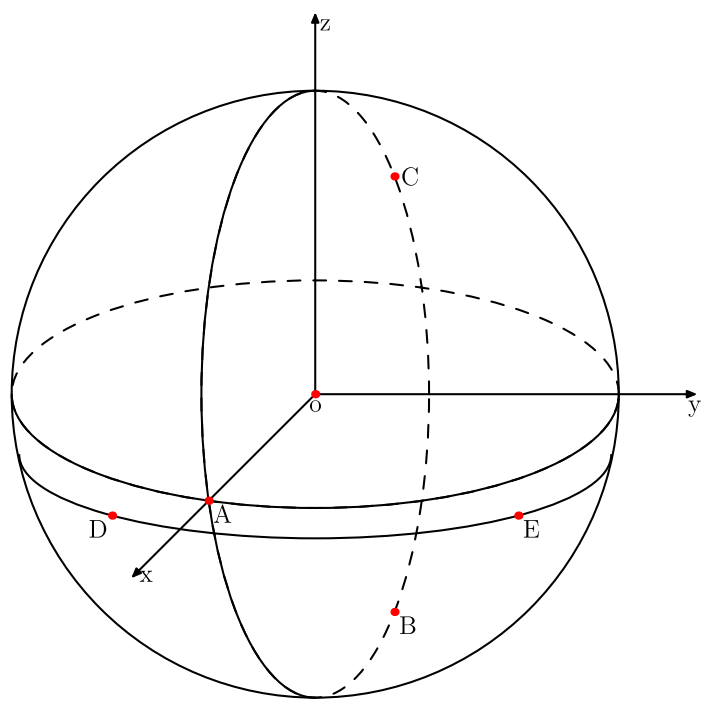

as shown in Fig. 3. The corresponding $\Theta$ for this distribution is

$$
\Theta_{p}=\left(-2 \omega_{1}, \frac{\pi}{2}-\omega_{1}, \pi, \omega_{2},-\omega_{3}, \omega_{2}, \omega_{3}\right) .
$$

We have

$$
f\left(\Theta_{p}\right) \approx 15.67482117
$$

\subsection{Neighborhood of $\Theta_{p}$}

Similarly as in Sect. 4.2, we introduce a perturbation $\left[-\frac{\pi}{791}, \frac{\pi}{791}\right]$ on the coordinates of $\Theta_{p}$, and finally obtain a rectangular domain $\mathcal{D}_{p}$.

The interval Hessian matrix $\mathcal{W}$ of $f$ over $\mathcal{D}_{p}$ can be calculated by interval computation. Through Theorem 4.4, we can judge that $\mathcal{W}$ satisfies $\mathcal{W} \npreceq 0$. So by Theorem 4.6, we know that $f$ cannot attain the maximum in $\stackrel{\circ}{p}_{p}$, i.e.,

Proposition 5.1 There exists no maximum point of $f$ in $\stackrel{\circ}{\mathcal{D}}_{p}$.

\section{The Other Domain}

In this section, we will analyze $f(\Theta)$ for $\Theta \in\left(\mathcal{D}^{(1)} \cup \mathcal{D}^{(2)}\right) \backslash\left(\stackrel{\circ}{\mathcal{D}}_{b p} \cup \stackrel{\circ}{\mathcal{D}}_{p}\right)$, and prove that $\Theta_{b p}$ is the unique global maximum point of $f$ in $\mathcal{D}^{(1)} \cup \mathcal{D}^{(2)}$ (without considering spherical symmetry). 


\subsection{Verification Criterions}

We divide $\left(\mathcal{D}^{(1)} \cup \mathcal{D}^{(2)}\right) \backslash\left(\stackrel{\mathcal{D}}{b p}_{b} \cup \mathcal{D}_{p}\right)$ into small rectangular domains, and verify these domains one by one. If all points in a rectangular domain $\mathcal{D}_{0}$ satisfy one of following conditions:

1. (By Assumption 3.2) $C$ is below $E$

2. (By Assumption 3.4) $A, B, C, D, E$ are in the same half sphere

3. (By Assumption 3.5) The distance between some two points of $A, B, C, D, E$ is less than $\frac{2}{15}$

4. (By Assumption 3.1) $A B$ is not the second largest distance in all 10 distances

5. $f(\Theta)<f\left(\Theta_{b p}\right)$

6. (By Theorem 3.2) One of following equations

$$
\frac{x_{q}}{x_{p}}=\frac{y_{q}}{y_{p}}=\frac{z_{q}}{z_{p}}, \quad p \in\{A, B, C, D, E\}
$$

is not satisfied for all $\Theta \in \mathcal{D}_{0}$, where $q=\sum_{i=1}^{4}\left(p-p_{i}\right) /\left|p-p_{i}\right|$

7. (By Proposition 3.3) A partial derivative of $f$ does not change signs in this domain

then we can exclude this domain. Indeed, if Conditions 1 or 4 are satisfied, each point in $\mathcal{D}_{0}$ is spherically symmetric to some point in $\left(\mathcal{D}^{(1)} \cup \mathcal{D}^{(2)}\right) \backslash \mathcal{D}_{0}$; if Conditions 3,6 or 7 are satisfied, points in $\mathcal{D}_{0}$ cannot be local maximum points of $f$ in $\mathcal{D}^{(1)} \cup \mathcal{D}^{(2)}$; if Conditions 2 or 5 are satisfied, points in $\mathcal{D}_{0}$ cannot be global maximum points of $f$ in $\mathcal{D}^{(1)} \cup \mathcal{D}^{(2)}$.

To check a condition for a rectangular domain, we first construct corresponding elementary functions which should be greater than, less than, or not equal to zero over this domain. Then through the interval computation introduced in Sect. 4.1.1, we can obtain intervals containing ranges of function values over the domain, as we do in Example 4.1, which, in fact, has checked Condition 5 for the rectangular domain $\mathcal{D}_{1}^{(2)}$. If the intervals do not contain zero, then we could make a judgement whether the condition is satisfied for the domain.

For example, in order to verify whether each point in a rectangular domain $\mathcal{D}_{0}$ satisfies Condition 6, we first set $p$ to be $A$, whose Cartesian coordinates are $(1,0,0)$. From (6.1), we construct functions as follows

$$
\begin{aligned}
& g_{1}(\Theta) \triangleq x_{q} y_{p}-x_{p} y_{q}=-y_{q}, \\
& g_{2}(\Theta) \triangleq x_{q} z_{p}-x_{p} z_{q}=-z_{q},
\end{aligned}
$$

where

$$
\begin{aligned}
y_{q}= & -\frac{\cos \left(\phi_{4}\right) \sin \left(\theta_{4}\right)}{\sqrt{2-2 \cos \left(\phi_{4}\right) \cos \left(\theta_{4}\right)}}-\frac{\cos \left(\phi_{3}\right) \sin \left(\theta_{3}\right)}{\sqrt{2-2 \cos \left(\phi_{3}\right) \cos \left(\theta_{3}\right)}} \\
& -\frac{\cos \left(\phi_{2}\right) \sin \left(\theta_{2}\right)}{\sqrt{2-2 \cos \left(\phi_{2}\right) \cos \left(\theta_{2}\right)}},
\end{aligned}
$$


and

$$
\begin{aligned}
z_{q}= & -\frac{\sin \left(\phi_{4}\right)}{\sqrt{2-2 \cos \left(\phi_{4}\right) \cos \left(\theta_{4}\right)}}-\frac{\sin \left(\phi_{3}\right)}{\sqrt{2-2 \cos \left(\phi_{3}\right) \cos \left(\theta_{3}\right)}} \\
& -\frac{\sin \left(\phi_{2}\right)}{\sqrt{2-2 \cos \left(\phi_{2}\right) \cos \left(\theta_{2}\right)}}-\frac{\sin \left(\phi_{1}\right)}{\sqrt{2 \cos \left(\phi_{1}\right)+2}} .
\end{aligned}
$$

Denote by $\tilde{g}_{i}$ the expanded mapping of $g_{i}$, as defined in (4.4), and let $X_{i}=\tilde{g}_{i}\left(\mathcal{D}_{0}\right)$, $i=1,2$. If 0 is not contained in $X_{1}$ or $X_{2}$, then (6.1) is not true for all $\Theta \in \mathcal{D}_{0}$, thus Condition 6 is satisfied. Similar steps can be taken when $p$ is set to be $B, C, D$, or $E$.

We choose appropriate verification criterions and the verification order for a domain to be verified. If verifications are not successful, we subdivide the interval with maximal width into two intervals of equal width, and verify the two subdomains recursively. We set a positive number, if the largest interval width of a domain we get in the above process is less than this number, we stop subdividing this domain, and record it. The recorded domains may contain coordinates corresponding to distributions of points with larger distance sums then $f\left(\Theta_{b p}\right)$. This process terminates when all subdomains have been verified or recorded.

The detailed algorithm is described in Appendix.

\subsection{Subdivision Process}

If the size of a domain is too large, the recursive verification process may take too much memory, thus reducing the efficiency of the algorithm. We first need to subdivide $\mathcal{D}^{(1)}$ and $\mathcal{D}^{(2)}$ into domains of appropriate sizes. After some experiments, we finally subdivide them the way that each interval of them is trisected, so we get $3^{7}=2187$ subdomains for each. Denote them respectively by

$$
\mathcal{D}_{1}^{(1)}, \mathcal{D}_{2}^{(1)}, \ldots, \mathcal{D}_{2187}^{(1)}
$$

and

$$
\mathcal{D}_{1}^{(2)}, \mathcal{D}_{2}^{(2)}, \ldots, \mathcal{D}_{2187}^{(2)}
$$

If some of these subdomains are difficult to verify successfully, we can again subdivide them the same way. Actually, the following domains need to be subdivided again:

$$
\begin{aligned}
& \mathcal{D}_{62}^{(1)}, \mathcal{D}_{158}^{(1)}, \mathcal{D}_{239}^{(1)}, \mathcal{D}_{863}^{(1)}, \mathcal{D}_{1102}^{(1)}, \mathcal{D}_{1105}^{(1)}, \mathcal{D}_{1106}^{(1)}, \mathcal{D}_{2114}^{(1)}, \mathcal{D}_{2132}^{(1)}, \\
& \mathcal{D}_{1105-1101}^{(1)}, \mathcal{D}_{1106-834}^{(1)}, \mathcal{D}_{1106-861}^{(1)}, \mathcal{D}_{1106-1099}^{(1)}, \mathcal{D}_{1106-1100}^{(1)}, \\
& \mathcal{D}_{1105-1101-1100}^{(1)}, \mathcal{D}_{1106-834-725}^{(1)}, \mathcal{D}_{1106-834-726}^{(1)}, \\
& \mathcal{D}_{1106-834-725-1752}^{(1)}, \mathcal{D}_{1106-834-726-1507}^{(1)}, \mathcal{D}_{1106-834-726-1750}^{(1)},
\end{aligned}
$$


where $\mathcal{D}_{1105-1101}^{(1)}$ denotes the 1101 st subdomain in all 2187 subdomains of $\mathcal{D}_{1105}^{(1)}$, other similar notations are understood the same way.

\subsection{Verification Result}

The Maple Package fivepoints implements algorithms described in the above sections. The following is verification time for various domains (may differ on different computers, it is the time used by computers with Pentium IV 3.0 GHz CPU, and $1 \mathrm{~GB}$ RAM):

1. Time used to verify domain $\mathcal{D}^{(1)} \backslash\left(\mathcal{D}_{b p} \cup \stackrel{\circ}{\mathcal{D}}_{p}\right): 782534.203$ seconds.

2. Time used to verify domain $\mathcal{D}^{(2)} \backslash\left(\stackrel{\circ}{\mathcal{D}}_{b p} \cup \stackrel{\circ}{\mathcal{D}}_{p}\right): 8797.600$ seconds.

3. Total time: 791331.803 seconds.

In the end, no domain has been recorded.

\subsection{Conclusion}

Denoted by $\mathcal{E}_{i}, i=1, \ldots, 7$, respectively, the unions of domains verified successfully through the 7 criterions in Sect. 6.1. Note that if a domain satisfies one condition, then the domain is excluded, and no other condition needs to be tested for this domain. Thus $\mathcal{E}_{1}, \ldots, \mathcal{E}_{7}$ have no common interior points and

$$
\left(\bigcup_{i=1}^{7} \mathcal{E}_{i}\right) \cup \stackrel{\circ}{\mathcal{D}}_{b p} \cup \stackrel{\circ}{\mathcal{D}}_{p}=\mathcal{D}^{(1)} \cup \mathcal{D}^{(2)} \text {. }
$$

Moreover,

1. Points in $\mathcal{E}_{S} \triangleq \mathcal{E}_{1} \cup \mathcal{E}_{4}$ are spherically symmetric to some points in $\left(\mathcal{D}^{(1)} \cup\right.$ $\left.\mathcal{D}^{(2)}\right) \backslash \mathcal{E}_{S}$.

2. Points in $\mathcal{E}_{G} \triangleq \mathcal{E}_{2} \cup \mathcal{E}_{5}$ cannot be global maximum points of $f$ in $\mathcal{D}^{(1)} \cup \mathcal{D}^{(2)}$.

3. Points in $\mathcal{E}_{L} \triangleq \mathcal{E}_{3} \cup \mathcal{E}_{6} \cup \mathcal{E}_{7} \cup \mathcal{D}_{p}$ cannot be local maximum points of $f$ in $\mathcal{D}^{(1)} \cup$ $\mathcal{D}^{(2)}$.

Thus we have

$$
\mathcal{D}^{(1)} \cup \mathcal{D}^{(2)}=\mathcal{E}_{S} \cup \mathcal{E}_{G} \cup \mathcal{E}_{L} \cup \stackrel{\circ}{\mathcal{D}}_{b p}
$$

Since $f$ should have a global maximum point in $\left(\mathcal{D}^{(1)} \cup \mathcal{D}^{(2)}\right) \backslash \mathcal{E}_{S}$, and this point is not in $\mathcal{E}_{G}$ or $\mathcal{E}_{L}$, it must be in $\stackrel{\circ}{D}_{b p}$. From Proposition 4.1 , we can see that

Proposition 6.1 $\Theta_{b p}$ is the unique global maximum point of $f$ in $\left(\mathcal{D}^{(1)} \cup \mathcal{D}^{(2)}\right) \backslash \mathcal{E}_{S}$.

This, in fact, completes the proof of the conjecture about the $(5,3)$-problem for $\lambda=1$.

Acknowledgements Our sincere thanks are due to the two anonymous referees for providing constructive comments and help in improving the contents of this paper. 


\section{Appendix: Algorithm for checking domains contained in $\left(\mathcal{D}^{(1)} \cup \mathcal{D}^{(2)}\right) \backslash\left(\dot{\mathcal{D}}_{b p} \cup \dot{\mathcal{D}}_{p}\right)$}

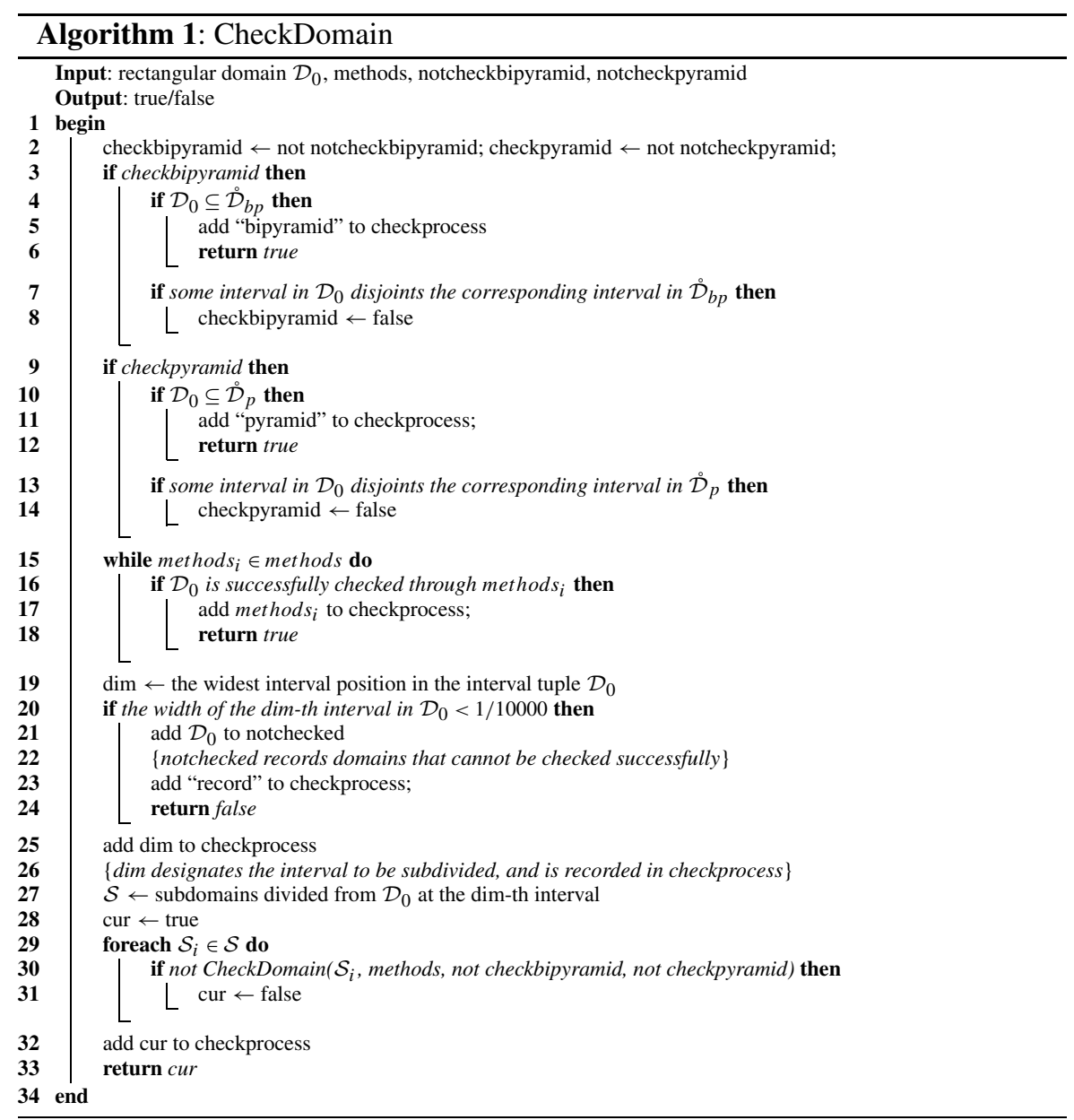

\section{References}

1. Alexander, R.: On the sum of distances between $n$ points on a sphere. Acta Math. Acad. Sci. Hung. 23(3-4), 443-448 (1972)

2. Alexander, R.: Generalized sums of distances. Pac. J. Math. 56(2), 297-304 (1975)

3. Alexander, R.: On the sum of distances between $n$ points on a sphere II. Acta Math. Acad. Sci. Hung. 23(3-4), 317-320 (1977)

4. Amdeberhan, T.: On sum of distances on a sphere. http://www.math.temple.edu/ tewodros/PAMS3. PDF

5. Andreev, N.N.: An extremal property of the icosahedron. East J. Approx. 2(4), 25-34 (1996)

6. Andreev, N.N., Yudin, V.A.: Problems of approximation theory in discrete geometry. In: Mathematical Research, vol. 107, pp. 19-32. Wiley-VCH, Berlin (1998) 
7. Beck, J.: Sums of distances between points on a sphere-an application of the theory of irregularities of distribution to discrete geometry. Mathematika 31, 33-41 (1984)

8. Berman, J., Hanes, K.: Optimizing the arrangement of points on the unit sphere. Math. Comput. 31(140), 1006-1008 (1977)

9. Chakerian, G.D., Klamkin, M.S.: Inequalities for sums of distances. Am. Math. Mon. 80(9), 10091017 (1973)

10. Cohn, H., Kumar, A.: Universally optimal distributions of points on spheres. J. Am. Math. Soc. 20, 99-147 (2007)

11. Croft, H.T., Falconer, K.J., Guy, R.K.: Unsolved Problems in Geometry. Springer, Berlin (1991)

12. Dragnev, P.D., Legg, D.A., Townsend, D.W.: Discrete logarithmic energy on the sphere. Pac. J. Math. 207(2), 345-357 (2002)

13. Fejes Tóth, L.: On the sum of distances determined by a pointset. Acta Math. Hung. 7(3-4), 397-401 (1956)

14. Fleming, W.: Functions of Several Variables, 2nd edn. Springer, Berlin (1987)

15. Füredi, Z.: The second and the third smallest distances on the sphere. J. Geom. 46, 55-65 (1993)

16. Golub, G.H., Van Loan, C.F.: Matrix Computation, 3th edn. Johns Hopkins University Press, Baltimore (1996)

17. Harman, G.: Sums of distances between points of a sphere. Int. J. Math. Math. Sci. 5(4), 707-714 (1982)

18. Hille, E.: Some geometric extremal problems. J. Aust. Math. Soc. 6, 122-128 (1966)

19. Jiang, M.: On the sum of distances along a circle. Discrete Math. 308(10), 2038-2045 (2008)

20. Katanforoush, A., Shahshahani, M.: Distributing points on the sphere, I. Exp. Math. 12(2), 199-209 (2003)

21. Kolushov, A.V., Yudin, V.A.: Extremal dispositions of points on the sphere. Anal. Math. 23(1), 25-34 (1997)

22. Pólya, G., Szegö, G.: Über den transfiniten Durchmesser (Kapazitätskonstante) von ebenen und räumlichen Punktmengen. J. Reine Angew. Math. 165, 4-49 (1931)

23. Rohn, J.: Positive definiteness and stability of interval matrices. SIAM J. Matrix Anal. Appl. 15(1), 175-184 (1994)

24. Saff, E.B., Kuijlaars, A.B.J.: Distributing many points on a sphere. Math. Intell. 19(1), 5-11 (1997)

25. Schwartz, R.E.: The 5-electron case of Thomson's problem. arXiv:1001.3702v5

26. Smale, S.: Mathematical problems for the next century. Math. Intell. 20(2), 7-15 (1998)

27. Stahl, V.: Interval methods for bounding the range of polynomials and solving systems of nonlinear equations. PhD thesis, University of Linz, Austria (1995)

28. Stolarsky, K.B.: Sums of distances between points on a sphere. Proc. Am. Math. Soc. 35(2), 547-549 (1972)

29. Stolarsky, K.B.: Sums of distances between points on a sphere, II. Proc. Am. Math. Soc. 41(2), 575$582(1973)$

30. Stolarsky, K.B.: Spherical distributions of $n$ points with maximal distance sums are well spaced. Proc. Am. Math. Soc. 48(1), 203-206 (1975)

31. Stolarsky, K.B.: The sum of the distances to certain pointsets on the unit circle. Pac. J. Math. 59(1), 241-251 (1975)

32. Stolarsky, K.B.: The sum of the distances to $n$ points on a sphere. Pac. J. Math. 57(2), 563-573 (1975) 